\title{
The relationship between body mass index, waist circumference and psoriatic arthritis in the Turkish population
}

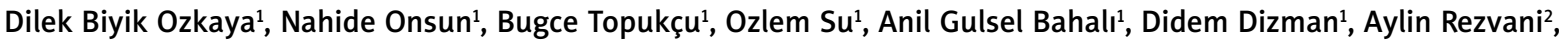 \\ Omer Uysal ${ }^{3}$
}

${ }^{1}$ Dermatoveneorology Department, Medical Faculty, Bezmialem Vakif University, Istanbul, Turkey ${ }^{2}$ Department of Physical Medicine and Rehabilitation, Medical Faculty, Bezmialem Vakif University, Istanbul, Turkey ${ }^{3}$ Department of Biostatistics and Medical Informatics, Medical Faculty, Bezmialem Vakif University, Istanbul, Turkey

Adv Dermatol Allergol 2016; XXXIII (3): 219-223

DOI: 10.5114/ada.2016.60615

\begin{abstract}
Introduction: Psoriasis is a chronic, immune-mediated inflammatory disease predominantly affecting the skin, with a complex aetiology. Recently it has been suggested that the chronic inflammation of psoriasis may cause metabolic and vascular disorders. The relationship between obesity and psoriatic arthritis (PsA) is not clear, and there are insufficient prospective studies addressing this subject.

Aim: To investigate the relationship between psoriatic arthritis, severity of psoriasis and obesity in the Turkish population.

Material and methods: Patient data from psoriasis outpatient clinics from February 2007 to July 2013 were reviewed retrospectively using the Psoriasis-Turkey (PSR-TR) registration system. Patients' age, onset age, body mass index (BMI), waist circumference, psoriasis area and severity index (PASI), and arthritis information were reviewed. In the outpatient clinics, patients who had joint pain consulted rheumatology clinics. The CASPAR criteria were used for the diagnosis of arthritis.

Results: A total of 443 males and 495 females enrolled in this study. The mean age of females was 43.9 years (18-93 years) and the mean age of males was 44.6 years (18-89 years). A total of 231 (25\%) patients had psoriatic arthritis. Investigation of the relationship between PASI, BMI, waist circumference (WC) and arthritis revealed a statistically significant relationship between each variable.

Conclusions: In this study we observed a relationship between PSA and high BMI, high WC and high PASI. Psoriatic arthritis is a chronic inflammatory disorder and a chronic inflammatory state induced by adiposity may lead to PsA.
\end{abstract}

Key words: psoriasis, psoriatic arthritis, body mass index, waist circumference.

\section{Introduction}

Psoriasis is a chronic, immune-mediated inflammatory disease predominantly affecting the skin, with a complex aetiology [1-3]. Recently it has been suggested that the chronic inflammation of psoriasis may cause metabolic and vascular disorders. Psoriatic arthritis (PsA), Crohn's disease, pustular diseases, metabolic syndrome, malignancies, respiratory system diseases, smoking, infectious diseases, quality of life impairment, depression and alcohol use are known comorbidities related to psoriasis $[4,5]$. Recent studies have shown that obesity is an inflammatory condition and that visceral fat behaves as an immune endocrine tissue $[6,7]$. It has been shown that adipokine produced from fat tissue has autocrine, paracrine and endocrine effects. Adipose tissue, in particular abdominal adiposity, acts as an endocrine organ secreting inflammatory mediators such as tumor necrosis factor (TNF)- $\alpha$, interleukin (IL) 6 , and adiponectin [8, 9].

Address for correspondence: Dilek Biyik Ozkaya, Dermatoveneorology Department, Medical Faculty, Bezmialem Vakif University, Adnan Menderes Bulvarı (Vatan Cad.) P.K.: 34093 Fatih, Istanbul, Turkey, phone: +90 5333506943, fax: 0 (212) 621 75 80,

e-mail: dilekozkaya@gmail.com

Received: 26.01.2015, accepted: 28.05.2015. 
The adipocytokines produced by fat tissue are TNF- $\alpha$, adiponectin, leptin, IL-6, plasminogen activator inhibitor type 1 (PAI-1) and IL-1 $[6,7]$. It has been shown that obesity may lead to chronic inflammation resulting from the increased production of TNF- $\alpha$, IL-6, PAI- 1 and C-reactive protein (CRP). Another effect of increased TNF- $\alpha, I L-6$ and PAI-1 levels is an increase in the visceral fat tissue $[6,7]$. Overproduction of inflammatory cytokines, such as TNF- $\alpha, I L-1, I L-6$, and IL-8, in adipose tissue is an important feature of obesity and may account for the pathogenesis of psoriasis [2, 10, 11].

Most known adipocytokines including adiponectin, resistin and visfatin contribute to insulin resistance and low grade inflammation. The cytokines adiponectin and resistin exert opposite effects on glucose metabolism. The cytokines not only play roles in metabolism but also are involved in development of chronic inflammation. Adipocytokines cause inflammation and tissue damage [12]. Leptin is the first described adipokine. Leptin has important immunoregulatory functions since it is involved in T cell proliferation, can induce $T$ helper type 1 immune reactions, and is involved in proliferation and activation of inflammatory cells such as monocytes and neutrophils [13]. Xue et al. suggested that leptin played a role in the exacerbation of PsA [13].

Although adiponectin plays a protective role in metabolic disorders such as obesity and insulin resistance, it is now well known that it also act as a pro-inflammatory factor in joints and may trigger matrix degeneration [12]. Adiponectin levels are found different in studies [12,13] Adiponectin has various isoforms and sometimes counteracting functions. Xue et al. found the level of total adiponectin significantly lower in patients with PSA than those in both healthy and psoriasis controls [13].

Chemerin enhanced the production of several proinflammatory cytokines (TNF- $\alpha, \mathrm{IL}-1 \beta, \mathrm{IL}-6$, and IL8) in human articular chondrocytes [13]. Xue et al. found chemerin significantly lower in the psoriasis (Ps) and PsA patients than in the healthy controls [13].

Studies have shown that omentin gene expression is altered by inflammatory states and obesity. Xue et al. found omentin significantly increased in patients with PsA compared with both healthy and psoriasis controls [13].

Corbetta et al. showed that increased serum resistin levels in untreated psoriasis patients were normalized after 1 and 3 months of acitretin therapy [14].

Resistin levels correlate with inflammation, joint destruction and levels of IL-1 receptor antagonists in women with rheumatoid arthritis (RA) [13].

Takahashi et al. showed that after treatment at the $24^{\text {th }}$ week the serum levels of adiponectin were significantly increased in all the treatment groups an in contrast, serum levels of leptin and resistin were significantly decreased (37 patients treated with infliximab, adalimumab, ustekinumab, NB-UVB) [15].
Fotiadou et al. found most of the cytokines IL-6, IL-8, IL-17A, IL-22, IL-23 and TNF- $\alpha$ elevated in the serum of psoriasis patients compared with controls. And they compared the patients with active disease and passive disease. According to their findings, IL-17A, IL-22 and IL-23 are strongly associated with the activity of the disease. In patients with stable disease they found these cytokines either absent or at very low concentrations [16]. In their study they confirmed that contribution of cytokines IL-6, IL-8, IL-17A, IL-22, IL-23 and TNF- $\alpha$ in the pathogenesis of psoriasis and indicates the possible role of IL-17, IL-22, and IL-23 in the active phase of the disease [16]. Benham et al. showed that Th17 and Th22 cells may have common as well as divergent roles in the pathogenesis of skin and joint disease in patients with Ps and PSA [17].

Many recent studies have focused on the comorbidities and the mechanisms of comorbidities that are associated with psoriasis. Psoriatic arthritis is a disease that may manifest different combinations of peripheral arthritis, dactylitis, spondylitis, and enthesitis [5]. Up to $40 \%$ of patients with psoriasis develop psoriatic arthritis with cartilage erosions, bone damage, and joint fusion [18]. The Caspar criteria are used for the diagnosis of psoriatic arthritis [19].

The relationship between obesity and PSA is not clear, and there are insufficient prospective studies addressing this subject. Some studies have reported a higher body mass index (BMI) and waist-hip ratio (WHR) in PSA patients compared to healthy controls [18, 20-22]. In this study we investigated the relationship between psoriatic arthritis, severity of psoriasis and obesity in the Turkish population.

\section{Material and methods}

Patient data from psoriasis outpatient clinics from February 2007 to July 2013 were reviewed retrospectively using the Psoriasis-Turkey (PSR-TR) registration system. Patients' age, onset age, BMI, waist circumference, PASI, and arthritis information were reviewed. In the outpatient clinics, patients who had joint pain consulted rheumatology clinics. The CASPAR criteria were used for the diagnosis of arthritis (Table 1) [19].

Body mass index and waist circumference were used for the diagnosis of obesity. Body mass index was calculated as the weight in kilograms ( $\mathrm{kg}$ ) divided by the square of the height in meters $\left(\mathrm{m}^{2}\right)$, and was therefore independent of age and sex. Body mass index $\leq 25 \mathrm{~kg} / \mathrm{m}^{2}$ was classified as normal, $25-30 \mathrm{~kg} / \mathrm{m}^{2}$ was classified as overweight, and $\mathrm{BMI} \geq 30 \mathrm{~kg} / \mathrm{m}^{2}$ was accepted as obesity.

A waist circumference $>88 \mathrm{~cm}$ in females and $>102 \mathrm{~cm}$ in males were accepted as high values, known to represent important cardiovascular risk factors [23].

We used the psoriasis area and severity index (PASI) to assess the degree of psoriasis severity. Patients who had a PASI value $\leq 5$ were considered to have mild pso- 
Table 1. The CASPAR criteria

To meet the CASPAR (CIASsification criteria for Psoriatic Arthritis) criteria, a patient must have inflammatory articular disease (joint, spine, or entheseal) with 3 points from the following 5 categories:

1. Evidence of current psoriasis, a personal history of psoriasis, or a family history of psoriasis. Current psoriasis is defined as psoriatic skin or scalp disease present today as judged by a rheumatologist or dermatologist. ${ }^{\dagger} \mathrm{A}$ personal history of psoriasis is defined as a history of psoriasis that may be obtained from a patient, family physician, dermatologist, rheumatologist, or other qualified health care provider. A family history of psoriasis is defined as a history of psoriasis in a first- or second-degree relative according to patient report.

2. Typical psoriatic nail dystrophy including onycholysis, pitting, and hyperkeratosis observed on current physical examination.

3. A negative test result for the presence of rheumatoid factor by any method except latex but preferably by enzyme-linked immunosorbent assay or nephelometry, according to the local laboratory reference range.

4. Either current dactylitis, defined as swelling of the entire digit, or a history of dactylitis recorded by a rheumatologist.

5. Radiographic evidence of juxta-articular new bone formation, appearing as ill-defined ossification near joint margins (but excluding osteophyte formation) on plain radiographs of the hand or foot.

riasis, a PASI value of $5-10$ corresponded to moderate psoriasis and PASI value $\geq 10$ indicated severe psoriasis.

\section{Statistical analysis}

For the statistical analysis, the $t$-test was used when comparing the averages of independent groups and the Pearson $\chi^{2}$ test was used when comparing the variance. In all tests a value of 0.05 was accepted as significant.

\section{Results}

A total of 443 males and 495 females enrolled in this study. The mean age of females was 43.9 years (18-93 years) and the mean age of males was 44.6 years (18-89 years). A total of 231 (24.6\%) patients had psoriatic arthritis. Investigation of the relationship between PASI, BMI, waist circumference (WC) and arthritis revealed a statistically significant relationship between each variable (BMI-arthritis t: $-4.971, p<0.001$; PASI-arthritis t: -5.865 , $p<0.001$; WC-arthritis t: -4.186, $p<0.001$ ) (Table 2).

There were 91 (40\%) males and 140 (60\%) females in the study presenting with arthritis, and 352 (49.8\%) males and 355 (50.2\%) females who did not have arthritis. A statistically significant correlation between arthritis and gender was found for females $(p=0.006)$.

Upon investigation of the correlation between BMI, PASI and waist circumference, the correlation between PASI-BMI was found to be statistically significant ( $p=$ $0.009, r=0.85$ ); the correlation between waist circumference and BMI was also statistically significant $(p<0.001$, $r=0.725)$.

Of the psoriasis patients, 433 individuals had a high waist circumference ( $\geq 88 \mathrm{~cm}$ in females, $\geq 102 \mathrm{~cm}$ in males), corresponding to 136 (31\%) male patients and 297 (59\%) female patients. The patients with a high waist circumference were found to have a higher risk of arthritis, and the risk was increased 1.26-fold if the patient was female (Table 3).
Table 2. The relationship between BMI, PASI, WC and arthritis

\begin{tabular}{llcc}
\hline Arthritis & & $\boldsymbol{N}$ & Mean \\
\hline \multirow{2}{*}{ BMI } & Arthritis(-) & 704 & 27.54 \\
\cline { 2 - 4 } & Arthritis(+) & 230 & 29.93 \\
\hline PASI & Arthritis(-) & 707 & 5.25 \\
\cline { 2 - 4 } & Arthritis(+) & 231 & 8.85 \\
\hline \multirow{2}{*}{ WC } & Arthritis(-) & 649 & 95.07 \\
\cline { 2 - 4 } & Arthritis(+) & 220 & 99.93 \\
\hline
\end{tabular}

Table 3. The relationship between sex and arthritis

\begin{tabular}{lcccc}
\hline \multirow{2}{*}{ Variable } & \multicolumn{3}{c}{ Gender } & Total \\
\cline { 2 - 4 } & & Male & Female & \\
\hline Arthritis(-) & $n$ & 352 & 355 & 707 \\
\cline { 2 - 4 } & $\%$ & 49.8 & 50.2 & 100 \\
\hline Arthritis(+) & $n$ & 91 & 140 & 231 \\
\cline { 2 - 4 } & $\%$ & 39.4 & 60.6 & 100 \\
\hline Total & $n$ & 443 & 495 & 938 \\
\cline { 2 - 4 } & $\%$ & 47.2 & 52.8 & 100 \\
\hline
\end{tabular}

\section{Discussion}

The prevalence of obesity continues to increase globally [3]. Psoriatic arthritis is a comorbidity of psoriasis; recent epidemiological studies show that PsA is more common than previously thought. Obesity is a medical condition in which excess body fat has accumulated to the extent that it may have adverse health effects, such as decreased longevity, diabetes mellitus, orthopaedic and respiratory disease and neoplasia [1]. Many studies have demonstrated the relationship between obesity and psoriasis [23-26]. However, the relationship between obesity and psoriatic arthritis is not clear and few prospective studies have addressed this research subject. 
Obesity is a significant risk factor for osteoarthritis at sites throughout the body; especially the knee [1], and some studies have shown a higher BMI and WHR in PSA patients compared to and healthy controls [20-22, 27].

The National Psoriasis Foundation survey (United States, 2002) reported that persistent joint pain or stiffness was found in $31 \%$ of patients with psoriasis, indicating that many patients may be unaware of their disease [28]. In this study, 24.6\% (231) of patients had psoriatic arthritis. However, these were patients with joint complaints and who were treated at rheumatology clinics. If we had investigated patients without joint complaints, this number might have been greater.

Psoriatic arthritis has a tremendous impact on health-related quality of life. Measures of pain and limitations related to emotional upset indicate that psoriatic arthritis may have more impact on the quality of patients' lives than RA [28]. Joint deformity and radiologically detectable damage have been demonstrated in at least $40 \%$ of those afflicted with psoriatic arthritis, and in some cases the disease may be as severe as RA. Many psoriasis patients initially visit dermatologists; each visit is an opportunity for a dermatologist to assess joint pain that may suggest the presence of psoriatic arthritis.

The problems associated with psoriasis include physical discomfort, disfigurement, and reduced quality of life, while the arthritic component adds to the burden with pain, swelling, stiffness, and reduced mobility and function. Physical signs of psoriatic arthritis include joint and entheseal swelling, "sausage" digits, and joint deformities. Radiologic manifestations include loss of joint space, bone and cartilage erosion, bony ankylosis, joint subluxation, periostitis, and subchondral cysts [28]. The erosive nature of psoriatic arthritis results in progressive deformities and restriction of functional ability.

The pathogenesis and causes of PsA are multifactorial in nature; genetic, environmental and immunologic factors play a major role in the development of this disease [22].

Li et al. [20] reported that the risk of PsA was moderately elevated with increasing BMI; compared with a BMI less than $25.0 \mathrm{~kg} / \mathrm{m}^{2}$, the risk of BMI 25.0-29.9, 30.0-34.9 and $35.0 \mathrm{~kg} / \mathrm{m}^{2}$ or greater increased to $1.83 ; 3.12$ and $6.46 \mathrm{~kg} / \mathrm{m}^{2}$, respectively. The authors found that participants with BMI of $35.0 \mathrm{~kg} / \mathrm{m}^{2}$ or greater were 2.98 -fold more likely to develop PsA. The results indicated a correlation between a markedly increased risk of PsA and BMI, weight change since early adulthood, waist and hip circumference, and WHR, both among total participants and among females with psoriasis [20].

Raychaudhuri et al. [21] reported that the prevalence of metabolic syndrome is high in patients with PsA. Patients with PSA appear to be at an increased risk of developing diabetes mellitus, arterial hypertension, dyslipidaemia, obesity and cardiac disease [15].
Tam et al. [22] reported a higher current BMI and WHR amongst PsA patients compared with healthy controls.

In this study we observed a relationship between PsA and high BMI, high WC and high PASI.

Setty et al. [2] examined the relationship between BMI, weight change, WC, hip circumference, waist-hip ratio, and incident psoriasis in 78,626 females over a 14year period (1991-2005) in the Nurses' Health Study II. The study found that multiple measures of adiposity, such as BMI, waist and hip circumference, waist-hip ratio, along with change in adiposity as assessed by weight gain since the age of 18 years, were substantial risk factors for the development of incident psoriasis. The risk increased with increasing levels of adiposity, demonstrating a strong, consistent dose-response relationship. In contrast, BMI of less than $21 \mathrm{~kg} / \mathrm{m}^{2}$ was associated with a lower risk of incident psoriasis [2].

Bhole et al. [18] investigated differences in BMI in PsA, Ps, RA and general population. They observed that individuals with PS, PSA and RA were at a greater risk of obesity than the general population, and that individuals with PsA had higher BMIs than those with Ps after adjusting for age, sex, smoking, Ps duration, PASI score, DMARDs, glucocorticoids, biologics and the MCS of the SF-36 health-related quality of life questionnaire. Bardazzi et al. [23] showed that patients who decreased their weight achieved a PASI score of 90 or 75 even if they did not respond at first.

Obesity plays a role in inflammation because fat acts as an endocrine tissue through the production of cytokines such as interleukin 6 and TNF- $\alpha$. Psoriatic arthritis is a chronic inflammatory disorder, and a chronic inflammatory state induced by adiposity may lead to PsA [20]. It is not understood how obesity increases the risk of PsA, whether by chronic inflammation or increased joint loading. In contrast, pain and inflammation may cause reduced physical activity in PSA patients, and this may increase the risk of obesity [18].

\section{Conclusions}

Psoriatic arthritis may affect quality of life more than other comorbidities of psoriasis. Treatment and protection is important, and dermatologists have a higher likelihood of seeing psoriasis patients prior to the onset of arthritis. Obesity not only affects the risk of psoriasis and psoriatic arthritis but also affects the treatment. Informing patients about the influence of obesity on their disease may reduce the risk of arthritis and other comorbidities of psoriasis.

\section{Conflict of interest}

The authors declare no conflict of interest. 


\section{References}

1. Kumar S, Han J, Li T, Qureshi AA. Obesity, waist circumference, weight change and the risk of psoriasis in US women. J Eur Acad Dermatol Venereol 2013; 27: 1293-8.

2. Setty AR, Curhan G, Choi HK. Obesity, waist circumference, weight change, and the risk of psoriasis in Women Nurses' Health Study II. Arch Intern Med 2007; 167: 1670-5.

3. Duarte GV, Oliveira Mde F, Cardoso TM, et al. Association between obesity measured by different parameters and severity of psoriasis. Int J Dermatol 2013; 52: 177-81.

4. Gülekon A, Ardişen E. Psoriasis and comorbidities. Turk Arch Dermatol 2008; 42 özel sayı: 2: 23-5.

5. Naldi L, Addis A, Chineti S, et al. Impact of body mass index and obesity on clinical response to systemic treatment for psoriasis. Dermatology 2008; 217: 365-73.

6. Puig L. Obesity and psoriasis: body weight and body mass index influence the response to biological treatment. JEADV 2011; 25: 1007-11.

7. Furuhashi M, Fucho R, Görgün ZC, et al. Adipocyte/macrophage fatty acid binding proteins contribute to metabolic deterioration through actions in both macrophages and adipocytes in mice. J Clin Invest 2008; 118: 2640-50.

8. Baran A, Flisiak I, Jaroszewicz J, Świderska M. Effect of psoriasis activity on serum adiponectin and leptin levels. Postep Derm Alergol 2015; 32: 101-6.

9. Sürücü HA, Aksoy N, Ozgöztas O, et al. Prolidase activity in chronic plaque psoriasis patients. Postep Derm Alergol 2015; 32: 82-7.

10. Schön MP, Boencke WH. Psoriasis. N Engl J Med 2005; 352: 1899-912.

11. Hamminga EA, van der Lely AJ, Neumann HA, Thio HB. Chronic inflammation in psoriasis and obesity: implications for therapy. Med Hypothese 2006; 67: 768-73.

12. Dikbas O, Tosun M, Bes C, et al. Serum levels of visfatin, resistin and adiponectin in patients with psoriatic arthritis and associations with disease severity. Int J Rheum Dis 2014 Sep 8. doi: 10.1111/1756-185X.12444.

13. Xue Y, Jiang L, Cheng Q, et al. Adipokines in psoriatic arthritis patients: the correlations with osteoclast precursors and bone erosions. PLoS One 2012; 7: e46740.

14. Corbetta S, Angioni R, Cattaneo A, et al. Effects of retinoid therapy on insulin sensitivity, lipid profile and circulating adipocytokines. Eur J Endocrinol 2006; 154: 83-6.

15. Takahashi H, Tsuji H, Ishida-Yamamoto A, lizuka H. Serum level of adiponectin increases and those of leptin and resistin decrease following the treatment of psoriasis. J Dermatol 2013; 40: 475-6.

16. Fotiadou C, Lazaridou E, Sotiriou E, et al. IL-17A, IL-22, and IL-23 as markers of psoriasis activity: a cross-sectional, hospital-based study. J Cutan Med Surg 2015; 19: 555-60.

17. Benham H, Norris P, Goodall J, et al. Th17 and Th22 cells in psoriatic arthritis and psoriasis. Arthritis Res Ther 2013; 15: R136.

18. Bhole VM, Choi HK, Burns LC, et al. Differences in body mass index among individuals with PsA, psoriasis, RA and the general population. Rheumatology (Oxford) 2012; 51: 552-6.

19. Taylor W, Gladman D, Helliwell P, et al. Classification criteria for psoriatic arthritis: development of new criteria from a large international study. Arthritis Rheum 2006; 54: 2665-73.

20.Li W, Han J, Qureshi AA. Obesity and risk of incident psoriatic arthritis in US women. Ann Rheum Dis 2012; 71: 1267-72.

21. Raychaudhuri SK, Chatterjee S, Nguyen C, et al. Increased prevalence of the metabolic syndrome in patients with psoriatic arthritis. Metab Syndr Relat Disord 2010; 8: 331-4.
22. Tam LS, Tomlinson B, Chu TT, et al. Cardiovascular risk profile of patients with psoriatic arthritis compared to controls the role of inflammation. Rheumatology 2008; 47: 718-23.

23. Bardazzi F, Balestri R, Baldi E, et al. Correlation between BMI and PASI in patients affected by moderate to severe psoriasis undergoing biological therapy. Dermatol Ther 2010; 1: 14-9.

24.Zhang C, Zhu KJ, Zheng HF, et al. The effect of overweight and obesity on psoriasis patients in Chinese Han population: a hospital based study. JEADV 2011; 25: 87-91.

25. Madanagobalane S, Anandan S. Prevalence of metabolic syndrome in south Indian patients with psoriasis vulgaris and the relation between disease severity and metabolic syndrome: a hospital-based case-control study. Indian J Dermatol 2012; 57: 353-7.

26. Duarte VG, Fatima M, Cardoso T, et al. Association between obesity measured by different parameters and severity of psoriasis. Int J Dermatol 2013; 52: 177-81.

27. Huang YH, Yang LC, Hui RY, et al. Relationships between obesity and the clinical severity of psoriasis in Taiwan. J Eur Acad Dermatol Venereol 2010; 24: 1035-9.

28. Mease P, Goffe BS. Diagnosis and treatment of psoriatic arthritis. J Am Acad Dermatol 2005; 52: 1-19. 